\title{
Una aplicación de la prueba chi cuadrado con SPSS
}

\begin{abstract}
RESUMEN
Se ilustra el uso del software estadístico SPSS aplicado a la prueba Chi Cuadrado para probar independencia entre dos variables. La aplicación se realiza en la evaluación del impacto generado en la plana docente de la Facultad de Ciencias Administrativas de la Universidad Nacional Federico Villarreal en relación al uso de algunas de las herramientas de las denominadas tecnologías de información y comunicación, en el proceso de formación profesional de Licenciados en Administración.
\end{abstract}

Palabras clave: SPSS, Prueba Chi Cuadrado, Tecnologías de información y comunicación (TIC)

ONE IMPLEMENTATION OF THE CHI SQUARE TEST WITH SPSS

\section{ABSTRACT}

Chi Cuadrado illustrates the use of the statistical software SPSS applied to the test to prove independence between two variables. The application carries out in the evaluation of the impact generated in the educational page of the Faculty of Administrative Sciences of the National University Federico Villarreal in relation to the use of some of the tools of the technologies called of information and communication in the process of formation profesional.

Keywords: SPSS, Test Chi Square, Information and communication technologies.

\section{INTRODUCCIÓN}

La Estadística es una herramienta usada en la investigación científica, teniendo como soporte diferentes programas informáticos, tales como el Statistical Package Social Science (SPSS), Stati Graph, Minitab, e incluso Microsoft Excel.

Según señalan Manzano y Perez (2002), investigadores de la Universidad de Sevilla (España), en un coloquio sobre el uso de aplicaciones informáticas para el análisis estadístico: "es inevitable y positivo el uso y la difusión de estas herramientas informáticas pero sin anteponer a la elevada capacidad de computación todas aquellas fases previas que están relacionadas con el correcto diseño de las investigaciones, y sin olvidar que toda investigación no puede ser realizada sin partir de un problema inicial al que se debe dar una respuesta".

Dentro de una larga lista de programas informáticos orientados a la Estadística, el SPSS destaca por su capacidad para procesar volúmenes de datos y por una interfase de fácil acceso al usuario. El Consejo Superior de Investigaciones de la Universidad Nacional Mayor de San Marcos (UNMSM) lo tiene como uno de los principales software de soporte y, por otro lado, su enseñanza y aplicación se realizan en las Unidades de Post Grado. Algo similar ocurre en otras universidades de la ciudad de Lima, tanto públicas como privadas.

El siglo XXI comprende una sociedad con cambios vertiginosos, donde todo cambia rápidamente al ritmo de los incesantes avances científicos y de acuerdo con las directrices del actual orden económico, de alcance mundial.

En este singular proceso de un mundo globalizado, las nuevas tecnologías de la información y las comunicaciones (TIC) tienen un papel muy importante, sin ellas simplemente se paralizarían muchas de las actividades cotidianas del hombre contemporáneo.

Marquez (2000), respecto a la importancia de las TIC en la educación moderna, resume las principales funcionalidades de las TIC en las entidades educativas: alfabetización digital de los estudiantes y profesores, acceso a la información, comunicación, gestión y proceso de datos, uso didáctico para facilitar los procesos de enseñanza y aprendizaje, relación entre profesores de diversos centros (a través de redes y comunidades virtuales), entre otras.

1 Magíster, Ingeniero Industrial, Docente de la UNMSM y de la UNFV.

E-mail: otinocog@unmsm.edu.pe 
La universidad pública peruana, pese a una serie de carencias estructurales, viene constantemente equipándose para afrontar un contexto cada vez más digitalizado. Las principales herramientas que adquieren ellas son computadoras personales, proyectores multimedia, ecrans, proyectores de transparencias, reproductores DVD, entre otros. Los responsables de la gestión académica y administrativa consideran en sus planes una mayor intensidad en el uso de las TIC, pero comprenden también que éste es un proceso que debe ir acompañado de la "alfabetización digital" de docentes y alumnos. Ello motiva el interés en abordar, en una primera evaluación, los impactos que han generado el uso de las herramientas ligadas a las TIC en la Facultad de Ciencias Administrativas de la Universidad Nacional Federico Villarreal (UNFV). El problema a ser develado consistió en estudiar el impacto del uso de las TIC en docentes de la mencionada Facultad

Una limitante del estudio fueron los formatos de solicitud de equipos Ilenados de manera deficiente y/o ilegible y que motivaron reajustes en el proceso de selección de la muestra.

\section{MATERIALES Y MÉTODOS}

El análisis del impacto de las TIC en docentes de la Facultad de Ciencias Administrativas de la UNFV presenta dos variables: por un lado, las TIC, expresadas en el tipo de equipo proporcionado por el laboratorio de Informática de dicha Facultad, y, por otro, la actividad docente desarrollada con el concurso de estas herramientas, expresada en el turno, Escuela Académica Profesional a la que conciernen las asignaturas involucradas y la categoría docente. Las dimensiones de ambas variables se midieron en escala nominal y corresponden a indicadores estadísticos cualitativos.

Para analizar la relación de dependencia y/o independencia entre dos variables cualitativas, se utiliza la prueba Chi Cuadrado de Independencia. Esta prueba permite determinar si existe una relación entre dos variables categóricas (cualitativas). Es necesario resaltar que esta prueba indica si existe o no una relación entre las variables, pero no señala el grado o el tipo de relación; es decir, no indica el porcentaje de influencia de una variable sobre la otra o la variable que causa la influencia.

La recopilación de información concerniente a estas variables comprendió los registros de atención a docentes y alumnos de la Facultad de Ciencias Administrativas, en el Laboratorio de Informática. Es preciso señalar que los equipos se concentran en el
Laboratorio de Informática y son solicitados por los delegados de las aulas que los requieran, atendiéndose en función del stock disponible de los mismos. El registro sistemático de este servicio ha permitido la aproximación a esta problemática universitaria.

Se realizó un corte transversal a junio de 2007 y se consideró como población objetivo 600 registros, correctamente llenados y correspondientes a una semana de atención; luego, considerando nivel de confianza $95 \%$, proporción $50 \%$ y margen de error $8 \%$ se obtuvo un tamaño de muestra de 120 registros.

De los 600 registros (población) contenidos en un archivo de datos de SPSS y correspondientes a la población definida, se seleccionaron aleatoriamente 120 registros, siguiendo la secuencia siguiente (Ver gráfico N. ${ }^{\circ 1)}$ :

Menú Datos / Seleccionar casos / Muestra aleatoria de casos / Muestra

\section{Gráfico N. ${ }^{\circ}$ 01: Cuadro de diálogo para seleccionar} muestra aleatoria en SPSS

\begin{tabular}{|l|}
\hline Seleccionar casos: Muestra aleatoria \\
\hline Tamaño de la muestra \\
OAproximadamente $\square$ \% de todos los casos \\
Exxactamente 120 casos de los grimeros $500 \quad$ casos \\
\\
Continuar Cancelar Ayuda
\end{tabular}

A partir de esta muestra aleatoria (120 registros) se procedió a efectuar el análisis estadístico; con la finalidad de verificar la asociación entre variables cualitativas, utilizando la prueba Chi Cuadrado, con un nivel de confianza de $95 \%$, y en uso del programa SPSS v. 14.0 en español.

\section{ANÁLISIS DE RESULTADOS}

Aspectos generales de la atenci n

La Facultad de Administración de la UNFV contaba en el 2007 con una población estudiantil de 1900 estudiantes, de los cuales 900 asisten en el turno noche, según información oficial contenida en su portal.

En el gráfico $\mathrm{N}^{\circ} 2$, se observa que la intensidad de uso de los equipos es mayor en el turno noche y menor en la tarde, hecho que se explica por la distribución de la población estudiantil en la facultad, en donde se registran. 


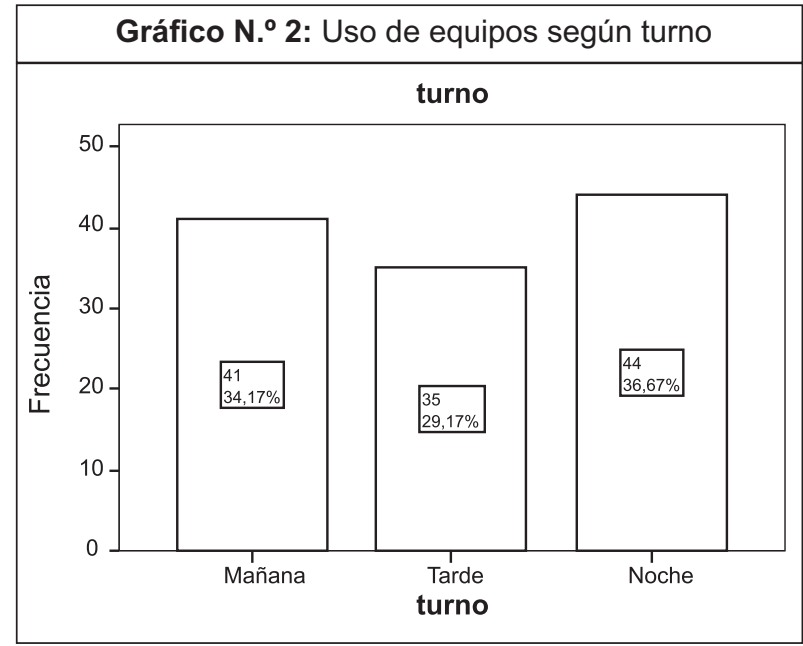

Fuente: Elaboración propia.

\begin{tabular}{|c|l|c|c|}
\hline \multicolumn{4}{|c|}{ Cuadro N. ${ }^{\circ}$ 1: Categoría Docente } \\
\hline \multirow{3}{*}{ Válidos } & & Frecuencia & Porcentaje \\
\hline & Auxiliar & 34 & 28,3 \\
\cline { 2 - 4 } & Asociado & 66 & 55,0 \\
\cline { 2 - 4 } & Principal & 20 & 16,7 \\
\cline { 2 - 4 } & Total & 120 & 100,0 \\
\hline
\end{tabular}

Fuente: Elaboración propia.

Según los datos del cuadro N. ${ }^{\circ} 1$, los usuarios docentes pertenecen, mayoritariamente, a la categoría asociados (55\%), seguido de los docentes auxiliares $(28,3 \%)$ y los principales $(16,7 \%)$

En el cuadro $\mathrm{N}^{\circ} 2$, se ilustra el nivel de atención con equipos de apoyo a la labor docente según turno. Mayoritariamente, se utilizan los proyectores multimedia, en los tres turnos.

El gráfico N.$^{\circ} 3$ ilustra el uso de los equipos según Escuela Profesional, el mismo que se corresponde con la densidad estudiantil de cada una de ellas en la Facultad.

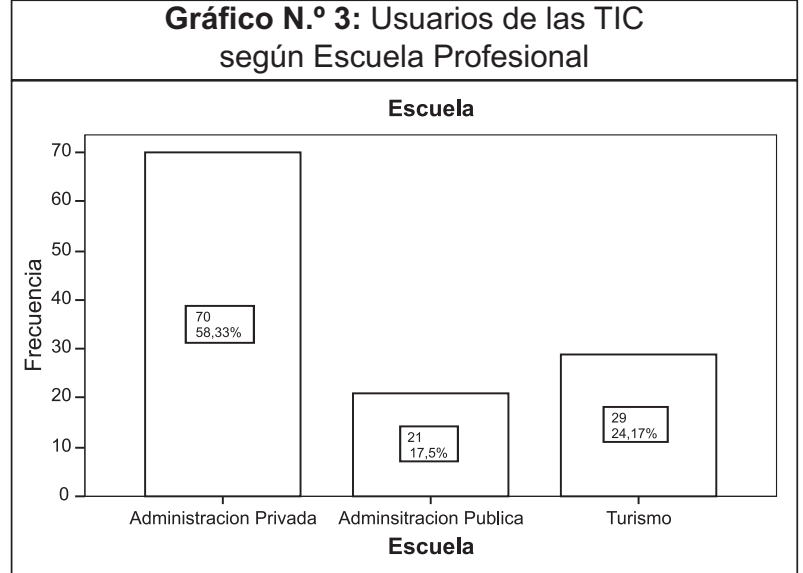

Fuente: Elaboración propia.

\section{APLICACIÓN DE SPSS PARA EVALUAR LA RELA- CIÓN CATEGORÍA DOCENTE Y USO DE EQUIPOS}

Para evaluar el impacto de las TIC en la actividad docente, se relacionó la categoría de los docentes (independiente) que hacen uso de estos equipos con el tipo de los mismos (dependiente), partiendo de la siguiente hipótesis:

Ho: las variables son independientes

$\mathrm{H} 1$ : las variables son dependientes

Para contrastar estas hipótesis, se utilizó la prueba de independencia del Chi-cuadrado, la misma parte del supuesto que las variables (Categoría docente y Uso de equipos) son independientes; es decir, que no existe ninguna relación entre ellas. El objetivo de esta prueba es contrastar la hipótesis mediante el nivel de significación, por lo que si el valor de la significación es mayor o igual que el Alfa (0.05), se acepta la hipótesis; pero si es menor, se rechaza.

La obtención de los resultados de la prueba Chi Cuadrado correspondiente a la verificación de estas hipótesis se realizó con el programa SPSS, siguiendo la secuencia:

Menú Analizar / Tablas / Tablas Personalizadas

\begin{tabular}{|l|r|r|r|r|}
\hline \multicolumn{5}{|c|}{ Cuadro N. ${ }^{\circ}$ 2: Tipo de equipo solicitado por turno académico } \\
\hline \multicolumn{1}{|c|}{ Equipo } & \multicolumn{1}{c|}{ turno } & & & \multicolumn{1}{c|}{ Total } \\
\hline & Mañana & \multicolumn{1}{c|}{ Tarde } & \multicolumn{1}{c|}{ Noche } & \\
\hline Retroproyector & 19 & 12 & 15 & 46 \\
\hline Proyector Multimedia & 22 & 23 & 29 & 74 \\
\hline Total & 41 & 35 & 44 & 120 \\
\hline
\end{tabular}

Fuente: Elaboración propia. 
Se ubicaron luego las variables señaladas en filas y columnas, tal como se ilustra en el gráfico $\mathrm{N} .{ }^{\circ} 4$, mostrado a continuación:

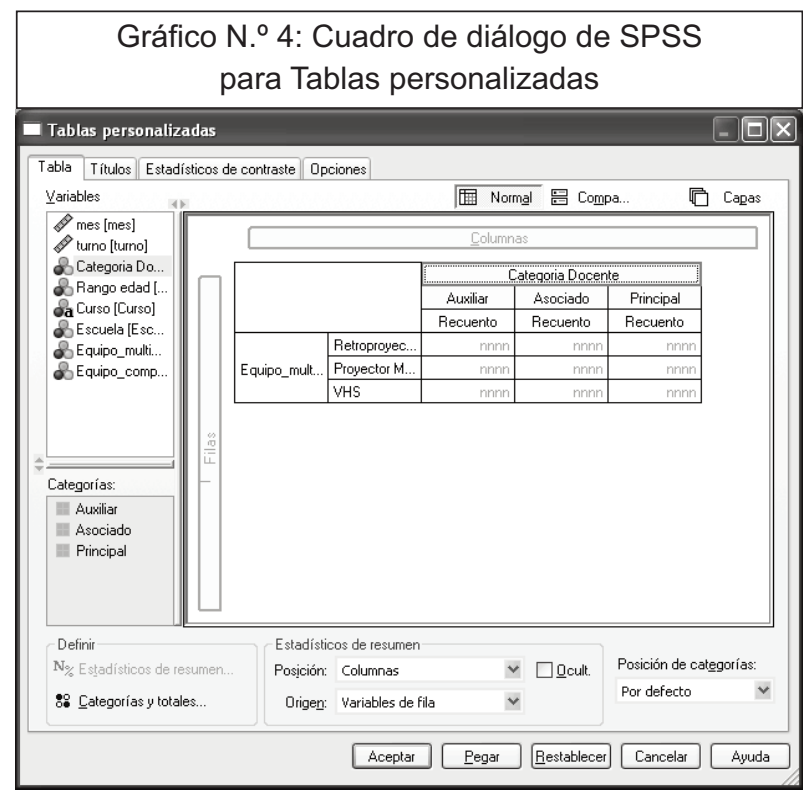

A continuación, se activó la pestaña Estadísticos de contraste, y dentro de ella se seleccionó Pruebas de Independencia (Chi Cuadrado), tal como se muestra en el gráfico N. ${ }^{\circ} 5$.

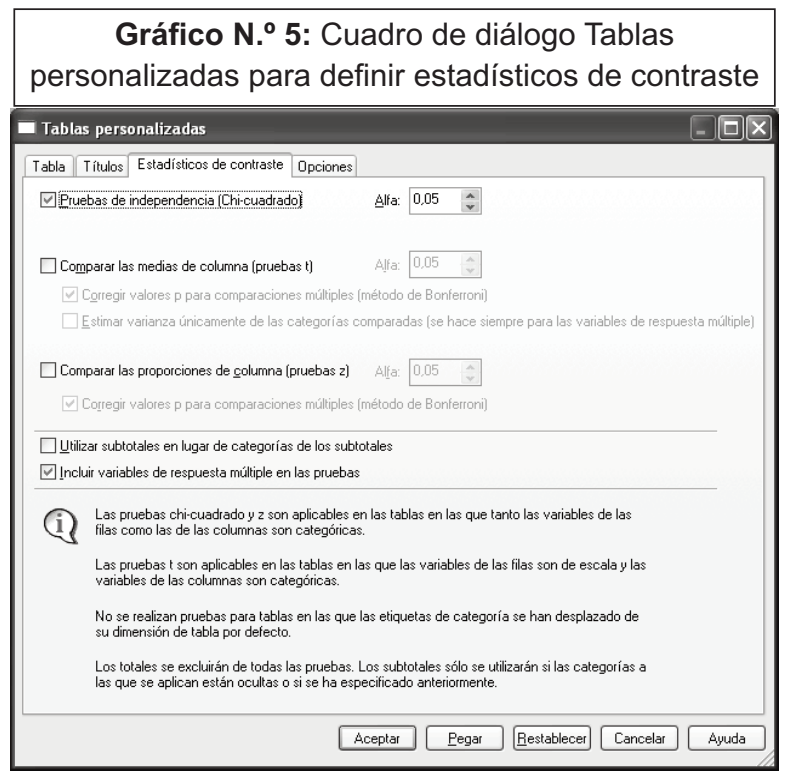

El Cuadro N. ${ }^{\circ} 3$ permite deducir que el uso del proyector multimedia, asociado estrechamente al concepto de las TIC, se usa mayoritariamente en las categorías Auxiliar y Asociado, y en paridad con el retroproyector en la categoría Principal:

\begin{tabular}{|c|c|c|c|c|}
\hline \multicolumn{5}{|c|}{$\begin{array}{c}\text { Cuadro } N^{\circ} 3: \text { Tipo de equipo solicitado según } \\
\text { categoría docente }\end{array}$} \\
\hline \multirow[t]{2}{*}{ Equipo } & \multicolumn{3}{|c|}{ Categoría docente } & \multirow[t]{2}{*}{ Total } \\
\hline & Auxiliar & Asociado & Principal & \\
\hline Retroproyector & 9 & 27 & 10 & 46 \\
\hline Proyector Multimedia & 25 & 39 & 10 & 74 \\
\hline Total & 34 & 66 & 20 & 120 \\
\hline
\end{tabular}

Fuente: Elaboración propia.

Junto con el cuadro anterior, aparecieron los siguientes indicadores de la inferencia, asociados a la prueba Chi Cuadrado):

Cuadro N. ${ }^{\circ}$ 4: Resultados Prueba Chi Cuadrado con SPSS

\begin{tabular}{|l|c|c|c|}
\hline & Valor & gl & $\begin{array}{c}\text { Sig. asintó- } \\
\text { tica (bilateral) }\end{array}$ \\
\hline $\begin{array}{l}\text { Chi-cuadrado de } \\
\text { Pearson N de } \\
\text { casos válidos }\end{array}$ & $\begin{array}{c}3,361 \\
120\end{array}$ & 2 &, 186 \\
\hline
\end{tabular}

El valor $p=0.186$, que se compara con el Alfa de significación (asumido como 5\%), es muy alto para rechazar Ho. Esta información denota que no hay indicios de una relación de dependencia entre ambas variables y, por lo tanto, se puede concluir que las variables no están relacionadas.

\section{RELACIÓN ESCUELA Y USO DE EQUIPOS}

Otra forma de aproximación al impacto de las TIC es relacionando la naturaleza de los estudios (Escuela) con el tipo de equipos que requieren ellas.

Se partió de las siguientes hipótesis: Ho: las variables son independientes $\mathrm{H} 1$ : las variables son dependientes

En el Cuadro N. ${ }^{\circ} 4$ se evidencia el uso preferente del proyector multimedia en todas las escuelas de la facultad, pero también refleja una mayor proporción de la Escuela de Administración Privada, en relación al uso de retroproyectores, en las escuelas de Administración Pública y Turismo. 


\begin{tabular}{|l|c|c|c|c|}
\hline \multicolumn{4}{|c|}{ Cuadro N. ${ }^{\circ}$ 5: Tipo de equipo solicitado según Escuela Profesional } \\
\hline \multirow{2}{*}{ Equipo } & \multicolumn{3}{|c|}{ Escuela } & Total \\
\cline { 2 - 5 } & $\begin{array}{c}\text { Administración } \\
\text { Privada }\end{array}$ & $\begin{array}{c}\text { Administración } \\
\text { Pública }\end{array}$ & Turismo & \\
\hline Retroproyector & 32 & 4 & 10 & 46 \\
\hline Proyector Multimedia & 38 & 17 & 19 & 74 \\
\hline Total & 70 & 21 & 29 & 120 \\
\hline
\end{tabular}

Fuente: Elaboración propia.

Cuadro N. ${ }^{\circ}$ 6: Resultados Prueba Chi Cuadrado con SPSS

\begin{tabular}{|l|c|c|c|}
\hline & Valor & gl & $\begin{array}{c}\text { Sig. asintótica } \\
\text { (bilateral) }\end{array}$ \\
\hline $\begin{array}{l}\text { Chi-cuadrado de } \\
\begin{array}{l}\text { PearsonN de } \\
\text { casos válidos }\end{array}\end{array}$ & $\begin{array}{c}5,099 \\
120\end{array}$ & 2 &, 042 \\
\hline
\end{tabular}

La prueba Chi Cuadrado efectuada con estos datos presenta el resultado ilustrado en el cuadro $\mathrm{N} .^{\circ} 5$, según el cual se rechaza la hipótesis nula ( $p=$ 0.042 menor que 0.05). Se deduce que ambas variables son dependientes, con un nivel de confianza de $95 \%$.

\section{CONCLUSIONES}

Hay un uso sostenido de equipos informáticos de apoyo a la labor docente en los tres turnos de atención de la facultad de Ciencias Administrativas.

Las tres escuelas muestran un uso predominante de los proyectores multimedia y en menor proporción de los retroproyectores.

El uso de las TIC es independiente de la categoría docente (auxiliar, asociado o principal).

La naturaleza de los estudios (escuela profesional) influye en la selección del tipo de ayuda de los equipos.
El uso de programas informáticos como el SPSS, simplifica el proceso de verificación de hipótesis.

\section{AGRADECIMIENTOS}

Al Magíster Carlos Tello Malpartida, Decano de la Facultad de Ciencias Administrativas de la UNFV, por haber permitido la realización del presente estudio, así como al personal administrativo de la Unidad de Informática de dicha entidad.

\section{REFERENCIAS BIBLIOGRÁFICAS}

1. Egocheaga, J. (2006). Apuntes de Investigación II: Métodos Cuantitativos. UNFV. Lima.

2. Mason, R.; Marchal, W. \& Lind, D. (2004). Estadística para Administración y Economistas. Alfaomega, Colombia.

3. Pere Marquez, G. (2000). Impacto de las TIC en Educación: Funciones y Limitaciones.

4. Tinoco, O. (2004). Aplicaciones Estadísticas con SPSS. Edit. Gutenberg, Lima.

5. Estadísticas de atención 2007 - Laboratorio de Informática. Facultad de Ciencias Administrativas, UNFV.

6. Manzano, V. y Perez, F. (2002). Análisis estadístico con aplicaciones informáticas: ¿ayuda o sustituye el razonamiento? Revista Metodología de Encuestas. Vol. 4, № 1, 128. 